Alessandro Serra, MD, $\mathrm{PhD}$

Margaret M. Skelly, PhD

Jonathan B. Jacobs, PhD

Mark F. Walker, MD

Jeffrey A. Cohen, MD
IMPROVEMENT OF INTERNUCLEAR

OPHTHALMOPARESIS IN MULTIPLE SCLEROSIS WITH DALFAMPRIDINE

Internuclear ophthalmoparesis (INO) in multiple sclerosis (MS) is due to demyelination of the medial longitudinal fasciculus (MLF) and provides an accessible model for studying consequences of raised body temperature and fatigue on central demyelination. ${ }^{1,2}$ Prompted by one of our patient's report of vision improvement after initiating dalfampridine, a potassium channel blocker prescribed for gait impairment, ${ }^{3}$ we measured this drug's effects on 3 patients with MS with bilateral INO. All showed changes in horizontal saccadic conjugacy consistent with improved transmission of the neural pulse responsible for adducting movements.

Classification of evidence. This study provides Class IV evidence that dalfampridine improves ocular adducting movements in patients with MS and bilateral INO.

Methods. Patients. We studied 3 men with MS (age 38-52) with chronic bilateral asymmetric INO who were taking dalfampridine for gait impairment. Patient 1 had diplopia and visual confusion when reading; patients 2 and 3 reported occasional visual confusion with head movements. Patients' visual acuity ranged from 20/20 to 20/30. Patient 1 had left eye exotropia; patients 2 and 3 had no strabismus. Besides bilateral INO, eye movement abnormalities included mild pendular nystagmus in both eyes in patient 3, bilateral gaze-evoked nystagmus in patients 2 and 3, choppy horizontal smooth pursuit in all patients, and abnormal convergence in patients 1 and 3. All patients gave written informed consent; the investigation was approved by the institutional review boards of the Louis Stokes VA Medical Center and the Cleveland Clinic and performed in accordance with the Declaration of Helsinki.

Eye movement recording. All patients withheld their dalfampridine $10 \mathrm{mg}$ morning dose until a baseline experimental session was completed. Patients made saccades in response to a small target light that jumped between 2 locations in the horizontal plane ( \pm 10 degrees of center position) at $1.0 \mathrm{~Hz}$ for 10 minutes. Binocular eye movements were recorded using the
EyeLink system (SR Research Ltd., Mississauga, Canada). A second session was repeated 3 hours after taking dalfampridine $10 \mathrm{mg}$ orally.

Data analysis. We used interactive programs written in MATLAB (The MathWorks, Inc., Natick, MA) to identify saccades using a $20 \mathrm{deg} / \mathrm{s}$ velocity threshold. We measured eye position and velocity and the abducting/adducting eye peak velocity ratio for each saccade. The latter aimed to compare the size of the saccadic pulse in the 2 eyes and in our laboratory the mean ratio $( \pm S D)$ is $1.04 \pm 0.08$ for healthy subjects. ${ }^{4}$

Results. All patients showed changes in horizontal saccadic conjugacy after dalfampridine, with patient 1 reporting improved quality of vision. Baseline hypometria of right eye adducting saccades and left eye dissociated nystagmus were clearly improved after dalfampridine for patient 1 (figure, A and B). Patient 1 had baseline left eye exotropia of about 10 degrees, which did not change after dalfampridine. Each patient showed significant decrease of saccadic abducting/adducting eye peak velocity ratios for the worst INO after dalfampridine, using the MannWhitney rank sum test $(p<0.05)$ (figure, C-E). For the milder INO, patients 1 and 2 showed significant increase of peak velocity ratio $(p<0.05)$ while patient 3 showed no significant change (figure, C-E). Before dalfampridine, patient 3 was only able to make recognizable saccades for the first 30 seconds, which provided 9-12 saccades in each direction for analysis, while for the rest of the recording he made saccades that were too slow to be detected by the minimum velocity threshold, which might have been due to an early fatigability effect. However, he was able to make recognizable saccades throughout the recording after dalfampridine.

Discussion. Stimulated by the report of improved vision of patient 1 while on dalfampridine, we evaluated its effect on INO-related gaze disconjugacy in 3 patients with MS. All showed substantial changes. Patients 1-3 showed improvement (i.e., decrease towards 1.0) of the abducting/adducting eye peak velocity ratio of saccades for the worst INO. However, the ratio was somewhat worse in the direction of the milder INO for patients 1 and 2 
A

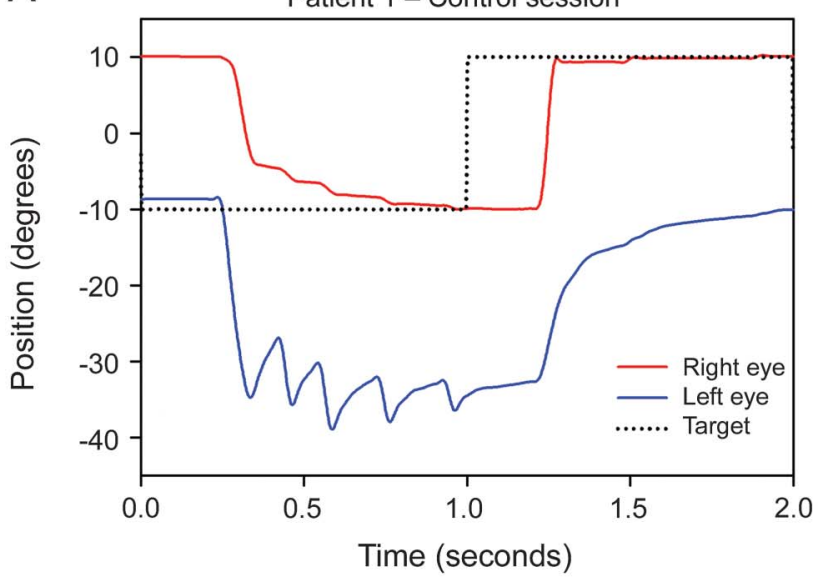

C

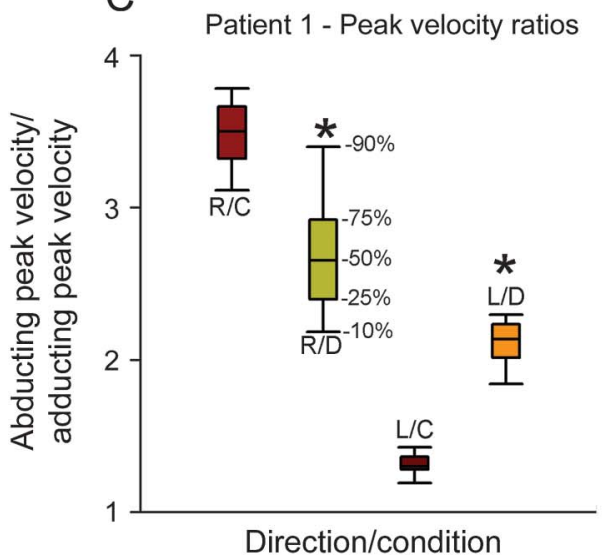

B

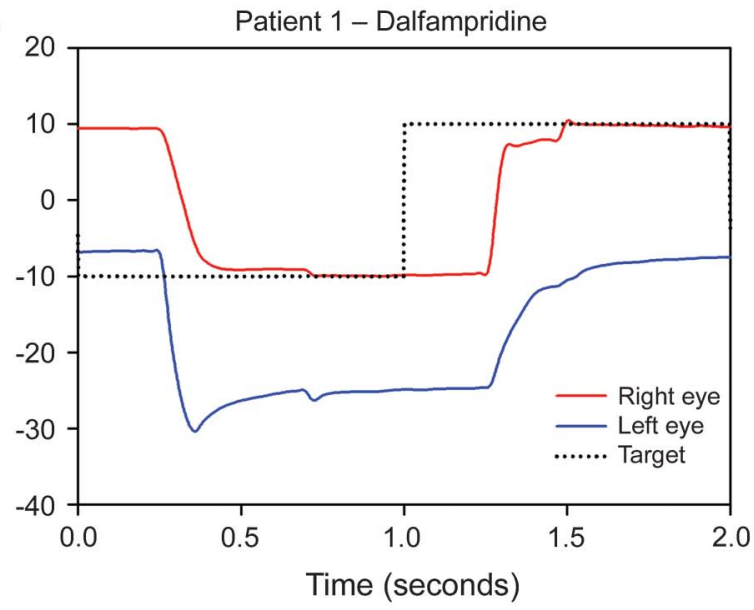

D

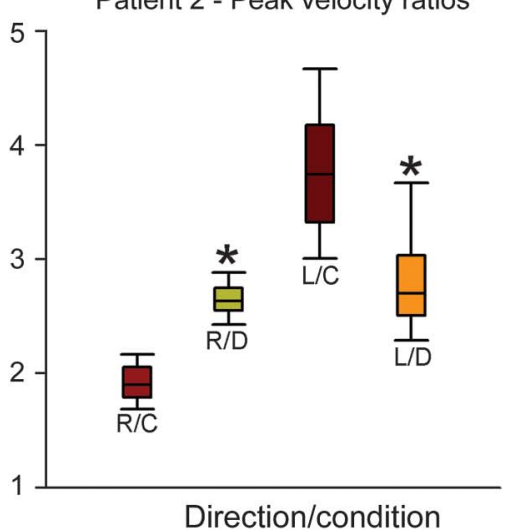

$E$

Patient 3 - Peak velocity ratios

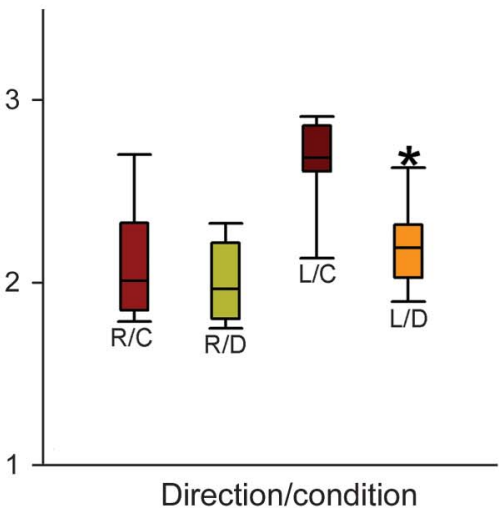

(A, B) Eye position records of a representative leftward and rightward saccade made by patient 1 before and after taking dalfampridine. Note that his baseline leftward saccade (A) is hypometric in the right, adducting eye and requires a series of saccades to reach the target with corresponding larger movements and nystagmus in the left, abducting eye; after taking dalfampridine (B), his right eye is almost able to reach the target with 1 saccade, a normal response, and the nystagmus in the left eye is decreased. Conversely, there is a mild decrease of the amplitude of his baseline left eye hypometric adducting saccade following dalfampridine $(A, B)$. Positive values indicate rightward movements; the initial leftward target jump occurs at the beginning of the record (not shown). (C-E) Abducting/adducting eye peak velocity ratios of the saccades for patients 1-3 before and after taking dalfampridine as box plots; percentiles are indicated in $\mathrm{C}$. In our laboratory the mean ratio $( \pm \mathrm{SD})$ is $1.04 \pm 0.08$ for healthy subjects. ${ }^{4} \mathrm{~L} / \mathrm{C}=$ leftward movements during control period; $L / D=$ leftward movements after dalfampridine; $R / C$ = rightward movements during control period; $R / D=$ rightward movements after dalfampridine. Note that in each case, the INO that is worse (larger ratio) at baseline improves with dalfampridine, whereas the milder INO shows a small ratio deterioration for patients 1 and 2 . Asterisks indicate a significant decrease of peak velocity ratio compared with the corresponding control condition.

(figure, C-E). For patient 1, improved visual quality was also likely due to improvement of adducting eye accuracy and reduction of abducting eye nystagmus (figure, $\mathrm{A}$ and $\mathrm{B}$ ). We interpret our observations as a possible reflection of enhanced neural conduction along the MLF due to dalfampridine.

As INO causes significant visual disability in patients with MS, which is often underestimated in the office, our findings of improved binocular conjugacy in the direction of the worst deficit suggest the need for a formal, controlled trial of dalfampridine in INO. Moreover, since INO can be used to model motor fatigue in $\mathrm{MS},{ }^{2}$ such a study might shed light on underlying poorly understood mechanisms of fatigue by assessing the effect of drugs such as dalfampridine on neural conduction along demyelinated pathways.

From The Daroff-Dell'Osso Laboratory (A.S., M.M.S., J.B.J., M.F.W.), Louis Stokes Veterans Affairs Medical Center; University Hospitals (A.S.); Case Western Reserve University (A.S., J.B.J., M.F.W.); and the Mellen Center for Multiple Sclerosis Treatment and Research (J.A.C.), Neurological Institute, Cleveland Clinic, Cleveland, $\mathrm{OH}$.

Presented in part at the 2013 Annual Meeting of the American Academy of Neurology.

Author contributions: A. Serra: study concept and design, acquisition, analysis, and interpretation of data, manuscript writing and critical revision. M. Skelly: acquisition and analysis of data. J. Jacobs: acquisition and analysis of data. $M$. Walker: critical revision of the manuscript. J. Cohen: critical revision of the manuscript.

Acknowledgment: The authors thank Drs. John Leigh, John Stahl, and Elliott Frohman for advice and encouragement. 
Study funding: Supported by the Office of Research and Development, Medical Research Service, Department of Veterans Affairs.

Disclosure: The authors report no disclosures relevant to the manuscript. Go to Neurology. org for full disclosures.

Received October 19, 2013. Accepted in final form March 12, 2014.

Correspondence to Dr. Serra: a.serra04@gmail.com

(C) 2014 American Academy of Neurology

1. Frohman TC, Davis SL, Frohman EM. Modeling the mechanisms of Uhthoffs phenomenon in MS patients with internuclear ophthalmoparesis. Ann NY Acad Sci 2011; 1233:313-319.

2. Matta M, Leigh RJ, Pugliatti $M$, et al. Using fast eye movements to study fatigue in multiple sclerosis. Neurology 2009;73:798-804.

3. Goodman AD, Brown TR, Edwards KR, et al. A phase 3 trial of extended release oral dalfampridine in multiple sclerosis. Ann Neurol 2010;68:494-502.

4. Chen AL, Ramat S, Serra A, et al. The role of the medial longitudinal fasciculus in horizontal gaze: tests of current hypotheses for saccade-vergence interactions. Exp Brain Res 2011;208:335-343.

\section{Minutes Pack a Punch}

\section{Neurology ${ }^{\circledR}$ Podcasts}

- Interviews with top experts on new clinical research in neurology

- Editorial comments on selected articles

- Convenient-listen during your commute, at your desk, or even at the gym

- On demand-it's there when you want it

- Fun and engaging

- New topic each week

- FREE

Listen now at www.aan.com/podcast

\section{WriteClick: Rapid Online Correspondence}

The editors encourage comments about recent articles through WriteClick:

Go to www.neurology.org and click on the "WriteClick" tab at the top of the page. Responses will be posted within 72 hours of submission.

Before using WriteClick, remember the following:

- WriteClick is restricted to comments about studies published in Neurology within the last eight weeks

- Read previously posted comments; redundant comments will not be posted

- Your submission must be 200 words or less and have a maximum of five references; reference one must be the article on which you are commenting

- You can include a maximum of five authors (including yourself) 